\title{
Modelica Stage Separation Dynamics Modeling for End-to-End Launch Vehicle Trajectory Simulations
}

\author{
Paul Acquatella B., and Matthias J. Reiner \\ German Aerospace Center (DLR), Institute of System Dynamics and Control \\ 82234 Wessling, Germany \\ paul.acquatella@dlr.de · matthias.reiner@dlr.de
}

\begin{abstract}
Stage separation dynamics modeling is a critical capability of future launchers preparatory studies. The development of stage separation frameworks integrable in end-to-end launch vehicle trajectory simulations have been presented in the relevant literature but none profiting from the object-oriented and equation-based acausal modeling properties of MoDELICA. The objective of this paper is therefore to present such an approach to this problematic. Based on the Constraint Force Equation (CFE) methodology, two case studies to evaluate the proposed approach are considered. Results demonstrate that the approach corresponds very well with the physics behind separation. In addition, we found easiness of implementation of the method within a single environment such as DYMOLA, demonstrating the benefits of an integrated approach.
\end{abstract}

\section{Introduction}

Stage separation dynamics modeling is a very challenging task and a critical capability that must be considered in the preparatory studies and development of next generation launchers [14, 16, 17]. The integration of such stage separation modeling into a single environment capable of end-to-end launch vehicle trajectory simulation is also a key technology to aim for.

The importance of such capability arises from the fact that after separation, the integrity of each stage must be kept in order to guarantee overall success of the space mission pursued. In this sense, the development of an integrated framework for analysis and simulation of stage separation is desired.

Early efforts on the subject of multi stage launch vehicle separation from the 60's and 70's are mainly from NASA studies [1, 2, 4] and their Program to Optimize Simulated Trajectories (POST) as a generalized trajectory simulation and optimization software [5], developed in partnership with the (then) Martin Marietta Corporation. Renewed interest in the subject in the 2000's led NASA's development of a stage separation conceptual separation tool, ConSep $[11,12,13,14]$; which is a MATLAB-based wrapper to the commercially available ADAMS solver, as its predecessor SepSim. However, being SepSim and ConSep dependent on the commercial software ADAMS, they have the disadvantage of not being easily integrable in a generic trajectory simulation software. This in turn eludes the capability of performing efficient end-to-end launch vehicle trajectory simulations. As a result, a generalized approach to stage separation problems of launch vehicles was developed [16]. The approach, coined as the Constraint Force Equation (CFE) methodology, was implemented into the Program to Optimize Simulated Trajectories II (POST2), the POST follow-up. Separation studies applied to real platforms such as the Hyper-X or the Space Shuttle can be found in $[18,10]$. The thesis [15] studies launcher separation analysis with OPENMODELICA but results in a tool (OMSep) which is only capable of input-output analyses at separation time, and not for generic launch vehicle trajectories.

As yet, an object-oriented and equation-based acausal modeling approach to stage separation dynamics integrable in end-to-end launch vehicle trajectory simulations is still missing. Such approach could potentially facilitate the integration of this and other capabilities within a single multi-physics environment such as DyMOLA.

The objective of this paper is therefore to present such an alternate approach to stage separation dynam- 
ics based on the CFE methodology using MODELICA [7, 8]. We do this by means of the following sub-objectives: We study first the modeling challenges of multi-stage launcher separation dynamics; then we present an approach based on CFE implemented in MODELICA; following, we provide two case studies for which we apply the method; and finally we present some results and discussion, outlining benefits and disadvantages.

\section{Modeling}

For the simulation of launch vehicle stage separation dynamics, it is necessary being able to model two bodies connected together according to properly-selected constraints prior to their physical separation; and at the release command of such constraints, their subsequent and independent flight motion must continue. This section presents the separation dynamics and the separation mechanisms modeling aspects.

\subsection{Separation dynamics}

We refer to separation dynamics in this paper the study of the effects of forces and torques of a two-body system during their physical separation.

Such separation dynamics modeling clearly exhibits discontinuities similar to those described by other phenomena such as switching, limiting, friction, etc. Modeling must deal with these problems in special ways since this kind of behavior is sensitive to numerical solution errors, initial condition calculation/propagation, and integration in general.

ModeliCA offers the possibility to implement a.o. several methods for such phenomena:

- Stop and restart: The complete system is simulated as a single body until separation time. Then the system is splitted into two bodies with independent states, and initial conditions are propagated accordingly. This solution however requires the split of two (or more) events.

- Regularization: This methodology consists on applying the constraint between the two bodies during their connected motion with a smooth but very stiff spring-damper system. This avoids the use of strict discrete or event behaviors. Such methodology is commonly used for simulation of friction, stiction, and other similar nonlinear behavior.
- Hybrid: This methodology consists on treating the simulation as a hybrid state machine where continuous and discontinuous behaviors are conditioned with data flows and proper transitions. This hybrid state machine framework is however complex to integrate in generic form for launch vehicle trajectory simulations.

- Constraint Force Equation (CFE) Methodology: The CFE methodology $[16,17,18]$ consists on computing internal constraint forces and moments on two bodies during their connected motion and their application as external forces and torques to each of them separately. On separation command, these internal forces are set to zero, and then each body carries their own flight motion separately.

Of these methods, particular interest due to its applicability and easiness of implementation is given to the CFE methodology, which is selected as the primary method for the follow up of this study.

\subsubsection{Constraint Force Equation Methodology}

The Constraint Force Equation (CFE) methodology $[16,17,18]$ is a highly intuitive method consisting in the computation of joint loads, namely internal forces and torques, caused by joint constraints; along with their application as external forces and torques on each body independently, see Figure 1.

The joint loads which constrain one body's motion relative to the other are dependent upon the external forces acting on each body as well as the type of joint. The net forces and torques on each body are therefore the sum of the usual external forces and torques plus the joint loads applied to each body as additional external forces and torques. In consequence, the CFE joint model simply augments the external loads of the system [17]. Quoting step by step [16, 17], the equa-
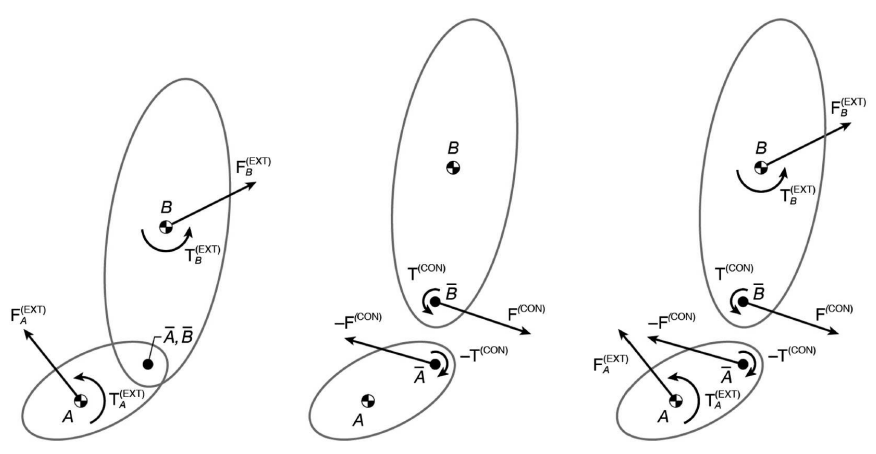

Figure 1: CFE diagram. Illustration credits: [16]. 
tions of constrained motion of two rigid bodies ( $A$ and $B$ ) connected by a single joint (point $\bar{A}$ in body $A$ and point $\bar{B}$ in body $B$ ) are as follows:

$$
\begin{gathered}
\mathbf{F}_{A}^{(e x t)}+\mathbf{F}_{A}^{(c o n)}=m_{A} \ddot{\mathbf{x}}_{A}, \\
\mathbf{T}_{A}^{(e x t)}+\rho_{A} \mathbf{F}_{A}^{(c o n)}+\mathbf{T}_{A}^{(c o n)}=\mathbf{I}_{A} \dot{\omega}_{A}+\omega_{A} \times \mathbf{I}_{A} \omega_{A}
\end{gathered}
$$

where $\rho_{A}$ is the position vector from the mass center of $A$ to point $\bar{A}$ of $A$ at which the constraint force is applied. Similarly for $B$ :

$$
\begin{gathered}
\mathbf{F}_{B}^{(e x t)}+\mathbf{F}_{B}^{(c o n)}=m_{B} \ddot{\mathbf{x}}_{B}, \\
\mathbf{T}_{B}^{(e x t)}+\rho_{B} \mathbf{F}_{B}^{(c o n)}+\mathbf{T}_{B}^{(c o n)}=\mathbf{I}_{B} \dot{\omega}_{B}+\omega_{B} \times \mathbf{I}_{B} \omega_{B} .
\end{gathered}
$$

There are so far 24 unknowns and 12 equations. Another set of six equations can be obtained from the law of action and reaction:

$$
\begin{gathered}
\mathbf{F}_{A}^{(c o n)}+\mathbf{F}_{B}^{(c o n)}=\mathbf{0} \\
\mathbf{T}_{A}^{(c o n)}+\mathbf{T}_{B}^{(c o n)}+\left(\mathbf{r}_{B}-\mathbf{r}_{A}\right) \times \mathbf{F}_{B}^{(c o n)}=\mathbf{0}
\end{gathered}
$$

where $\mathbf{r}_{A}=\mathbf{x}_{A}+\rho_{A}$ and $\mathbf{r}_{B}=\mathbf{x}_{B}+\rho_{B}$.

Six equations are missing. Worth noticing at this point, we only consider a single joint which constrain all six remaining degrees of freedom between the two bodies. This is because our focus is towards trajectory simulations and having multiple connections is not necessary unless when considering actuator sizing, sensitivity analyses, etc. In general, the CFE methodology allows to consider any type of joint which allows or not any specific relative motion between bodies; and redundancy of joints when necessary.

In this sense, for relative translation constraints and $\mathbf{e}$ being unit-vectors of the corresponding ( $A$ or $B$ ) bodyframe, it is required that:

$$
\left(\mathbf{r}_{B}-\mathbf{r}_{A}\right) \cdot \mathbf{e}_{A}=0
$$

meaning that the distance between the two points of a particular direction remain fixed. And finally, for relative rotations constraints, it is required that:

$$
\mathbf{e}_{A} \cdot \mathbf{e}_{B}=0
$$

meaning that three properly selected two-unit-vector sets must remain perpendicular.

Eqs. (7)-(8) would have to be differentiated twice with respect to time so that the resulting relationships involve the unknown accelerations and angular accelerations, thus finally being able to couple them with the equations of motion. In other words, the six missing equations are given by the following generalized constraint equations of the joint:

$$
\ddot{g}=0
$$

where $g$ represents either of the nondifferentiated constraints in Eqs. (7) and (8). As it will be demonstrated in the next section, the manual differentiation of Eqs. (7)-(8) and their coupling with the equations of motion can be avoided altogether by the MODELICA implementation since this is done automatically. The last important aspect of the CFE methodology relevant to this work is the accuracy of the joint loads solution, which is sensitive to computational error and initial joint missalignment [17]. To handle such concern, the CFE algorithm could feature a.o. a stabilization technique known as Baumgarte stabilization $[3,6,16]$. This particular stabilization technique consists on replacing the ODE given by Eq. (9) which allows perturbations to grow linearly with time, by the following asymptotically stable $\operatorname{ODE}(\eta>0)$ involving terms of the once differentiated and nondifferentiated forms of $g$ :

$$
\ddot{g}+2 \eta \dot{g}+\eta^{2} g=0
$$

however at the expense of more computational effort. Many other stabilization techniques [6] could be implemented; these other methods, and a guidance for selecting $\eta$ are however out of the scope of this paper.

\subsection{Physical modeling of multi-stage separa- tion mechanisms}

Separation mechanism refers in this proposal to a mechanical model (or device) that makes separation possible in simulation (or reality). Physical modeling refers in this context on the capability to model separation behaviour by considering first principles (kinematics, dynamics, mechanics, physics, etc.); and being able to get realistic insight from such models for other purposes such as actuator sizing, sensitivity analyses, control, optimization, etc.

Based on our internal DLR Space Systems Library, separation mechanism physical models of different complexity levels can be studied. Simplified models for preliminary and conceptual studies; and more detailed ones for engineering validation aspects. These varying degrees of complexity would be helpful in order to perform separation mechanics analyses and 
to assess the performance of the overall separation.

Configuration details of the separation mechanisms as well as their physical specifications must be provided to achieve more detailed and realistic models. Concerning the simple models, four variants have been studied:

- Linear charge (release device): The linear charge model performs ideal or benchmark separation between two bodies. This mechanism "cuts" the two-body system on command. It simulates (ideal) explosive release devices, clamps, diaphragms, or point-release devices such as explosive bolts.

- Bushing (separation impulse device): This model performs an impulsive reaction due to the release of a smooth but very stiff spring-damper system which keeps the two body system connected until separation command.

- Kick-off spring (separation impulse device): Same as before, the impulsive reaction due to the release of a spring-damper system simulates the proper transmission of forces and moments of the two-body system during separation. This model is implemented with the Constraint Force Equation (CFE) methodology. This element is combined with a release device to simulate a realistic kick-off spring mechanism.

- Generic (auxiliary devices): Other generic devices can be modeled in combination with the previous models, or with any other physical model from the library.

\section{ModeliCA implementation}

In this section, the ModeliCA implementation of separation mechanism models is presented. The challenges of this implementation strongly depends on the method selected as outlined in Section 2. Since the separation models in this work relies on a proper combination of the CFE methodology with physically-relevant elements, the implementation is not a straightforward application of existing MODELICA libraries; other aspects such as proper setup of initial conditions, state selection, modularity, and extendability are also challenging.

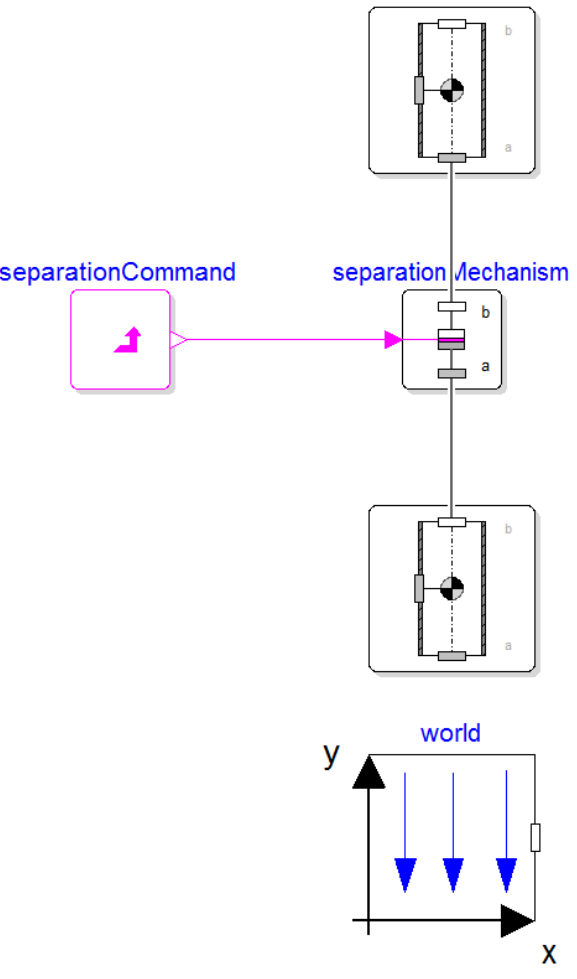

Figure 2: DyMOLA simulation layout consisting on a world model, two instances of rigid bodies, the separation mechanism model, and a boolean input for the separation command.

The baseline for the development of separation dynamics and separation mechanisms is the following partial mechanism model:

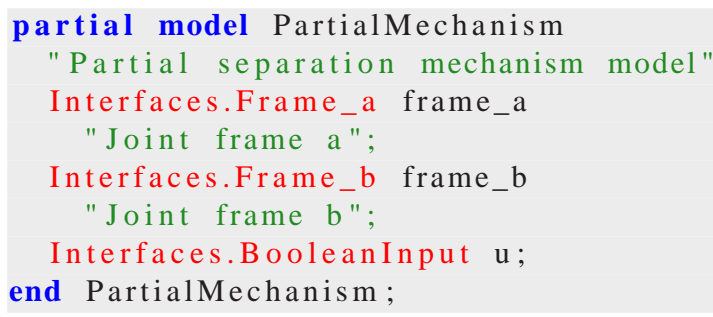

As shown in the code, the partial mechanism interface model consists of two frames to connect a two-body system, and a boolean input for the ignition or separation command. Such interface allows the use of several separation models depending on the desired level of complexity by using repleaceable instances. The approach here is bottom-up design, where the basis of separation dynamics simulation comes first from a single instance of a 'release device' mechanism.

In this work, a release mechanism model is implemented to simulate both a linear charge device com- 
monly used in launcher stage separation, where the forces and moments at separation are zero; and as a base model for the next level of complexity. In other words, for the implementation of a separation impulsive device, an instance of a release device providing the capabilities of joint motion until separation is required on top of another physical model providing the corresponding impulsive forces or moments at the time of separation. Therefore, increasing the functionality to the separation model will consist on adding impulsive devices or simply improving the physics behind the device in question.

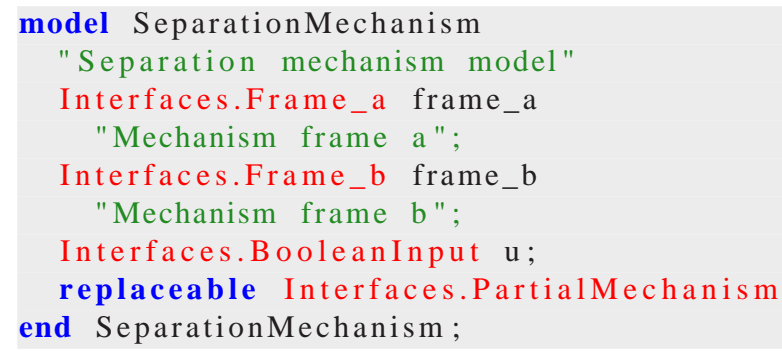

The implementation of the CFE procedure in MODELICA is as follows. The generalized constraint equations of the joint (9) have to be differentiated twice as explained before. Translational and rotational constraints at the joint are hence implemented as:

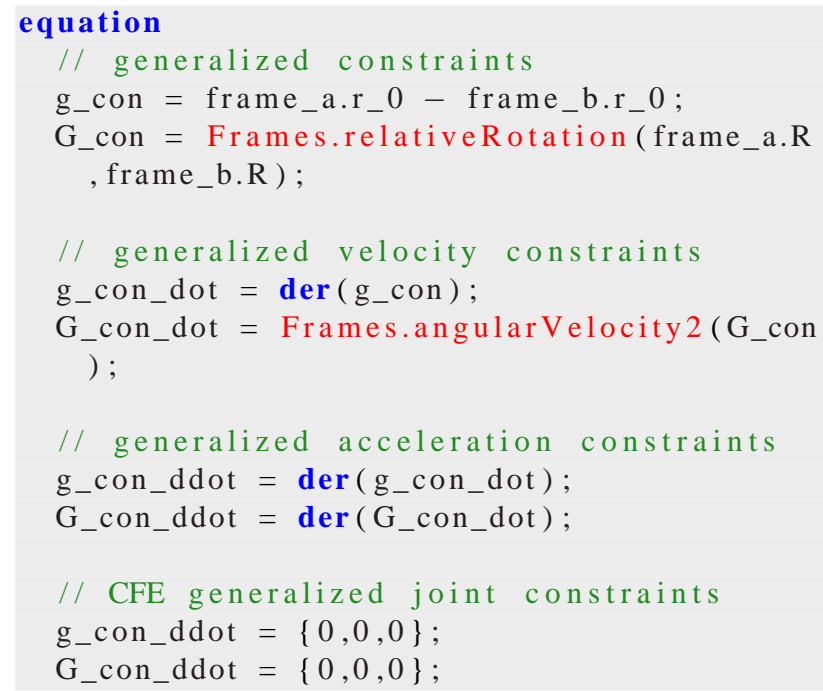

In short, we present briefly two of the main models developed in this work:

- Linear charge (separation release device): A release device is modeled by an instance of the SeparationMechanism model, called for instance linearCharge, which contains the partial interface outlined before, plus a switching mechanism between the CFE methodology and free body mo- tion.

- Kick-off spring (separation impulse device): An impulsive device is modeled by an instance of the SeparationMechanism model, called for instance kickOffSpring, which contains a linearCharge instance, plus a replaceable separationMechanism instance simulating the physics behind the impulsive device, such as a spring-damper system.

For a practical scenario to study, consider the trajectory phase of a generic launcher where the payload (Body $B$ - the satellite to be placed in orbit) is to be separated from the remaining launcher upper stage (Body $A$ - assuming a multi stage launcher). In this case, the problem consists of two bodies flying together under the effect of gravity in joint motion (the composite) up until separation is commanded. The separation command is usually given immediately after the shut down of the upper stage main engine. In this study however, we provide the separation command at any specified time. Figure 2 shows the DYMOLA simulation layout while Figure 3 shows a simulation of the physical setup of the case studies.

Initial conditions with respect to Earth-CenteredInertial (ECI) frame of the composite are given to Body $A$ as follows:

$$
\begin{aligned}
& \mathbf{x}_{A}(t=0)=\left[\begin{array}{c}
1.1378 \times 10^{7} \\
0 \\
0
\end{array}\right] \mathrm{m}, \\
& \mathbf{v}_{A}(t=0)=\left[\begin{array}{c}
0 \\
5.9188 \times 10^{3} \\
0
\end{array}\right] \mathrm{m} / \mathrm{s}
\end{aligned}
$$

and their translational and rotational dynamics are obtained from the rigid body model of the Modelica Multibody Library [9]. In the following section, we will study the separation dynamics implementation in ModELICA by means of two case studies: the first one considers the upper stage and payload (the composite) joint motion, while the second study considers the separation phase. For both cases, the forces due to gravity acceleration are obtained from the EGM96 model implemented in our internal DLR Space Systems Library. Both case studies are implemented in DyMOLA and the solution is computed using the DASSL solver with a tolerance of $1 e-7$. A smaller tolerance of this solver would increase significantly the resulting chattering when Baumgarte stabilization is used. 


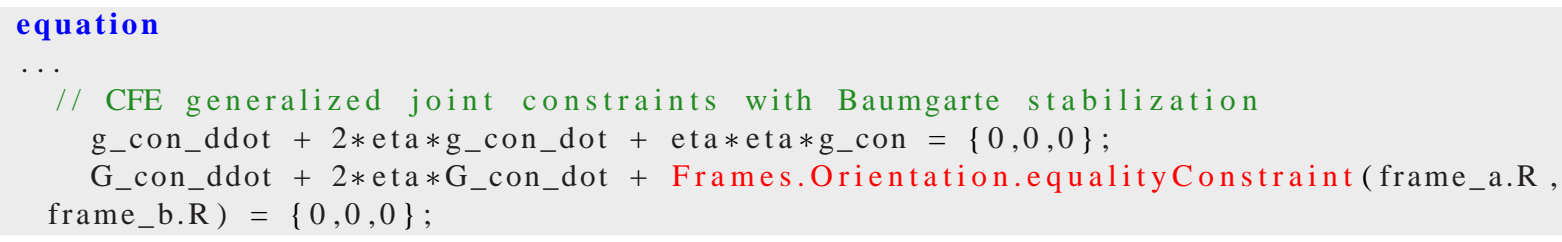

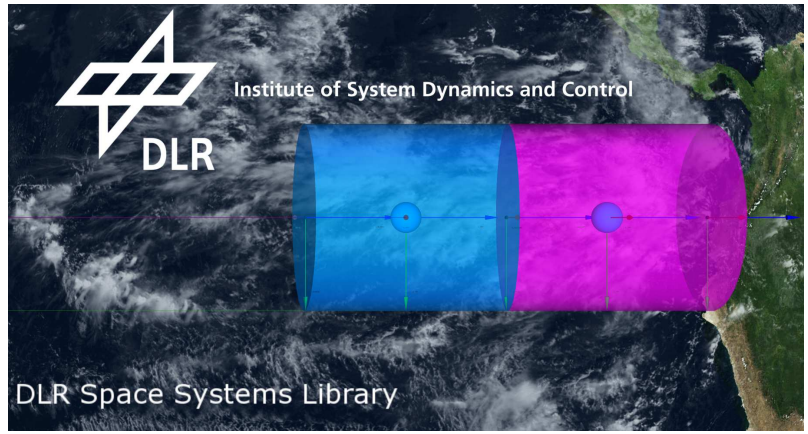

Figure 3: Simulation of the physical setup of the case studies.

Table 1: Mechanical properties of the two-body system.

\begin{tabular}{cccc}
\hline Property & Body $A$ & Body $B$ & Units \\
\hline Mass & 6000 & 1000 & $\mathrm{Kg}$ \\
$I_{11}$ & 23000 & 800 & $\mathrm{Kg} \cdot \mathrm{m}^{2}$ \\
$I_{22}$ & 23000 & 800 & $\mathrm{Kg} \cdot \mathrm{m}^{2}$ \\
$I_{33}$ & 18000 & 600 & $\mathrm{Kg} \cdot \mathrm{m}^{2}$ \\
$I_{21}=I_{31}=I_{32}$ & 0 & 0 & $\mathrm{Kg} \cdot \mathrm{m}^{2}$ \\
\hline
\end{tabular}

\subsection{Case study I: upper stage and payload (composite) joint motion}

The joint motion of the composite (Bodies $A$ and $B$, the upper stage and the payload respectively) is simulated for a total time of $2000 \mathrm{~s}$. During such motion, the MODELICA implementation of the CFE methodology is expected to derive automatically the joint constraint forces and torques such that the two-body system stays properly connected, with relative zero displacement. This case study therefore accounts for the validity of such implementation.

\subsection{Case study II: upper stage payload sepa- ration dynamics}

The upper stage payload separation is simulated in a practical scenario setup. It consists of a simulation of $20 \mathrm{~s}$, half of which is in connected or joint motion, and then at $t=10 \mathrm{~s}$, the ignition command for separation is given. At this point, a kick-off spring separation mechanism model is in charge of the dynamical separation between the bodies. The subsequent independent motion of each body is then expected. This case study therefore accounts for the applicability of the physical models of separation mechanisms implemented.

\section{Results and discussion}

As outlined in the last section, Case Study I accounts for the study of internal forces and torques of the composite joint motion during a given portion of its trajectory by means of the Constraint Force Methodology implemented in ModeliCA. During such joint motion, an important metric to assess the proposed method is the relative joint displacement between the two bodies when they are supposed to stay connected, as proposed and suggested by [17].

In this respect, Figure 4 presents the resulting constraint forces $\mathbf{f}[i]$ and torques $\operatorname{tau}[i]$ at the joint during the connected motion, in all ECI directions $i=x, y, z$, respectively; while Figure 5 presents the resulting relative joint position $\operatorname{rrel}[i]$ and the relative joint velocity $\operatorname{vrel}[i]$, in all ECI directions $i=x, y, z$, respectively.

Results shows that the corresponding joint constraint forces and torques, obtained automatically by MODELICA in order to satisfy the CFE methodology constraints successfully keeps the bodies properly connected (hence, the composite) during their connected flight motion. Such result is evidenced looking at the relative joint position and relative joint velocity between the two bodies, which are supposed to be zero during the connected flight. A clear disadvantage for long simulation periods of joint composite motion is the necessity to keep the drift within physical boundaries, hence requiring a stabilization method. Stability and accuracy of the solution, especially for large simulation times, are improved with the addition of the Baumgarte stabilization. Nevertheless at the expense of chattering as shown in Figures 4-(b), 4- 


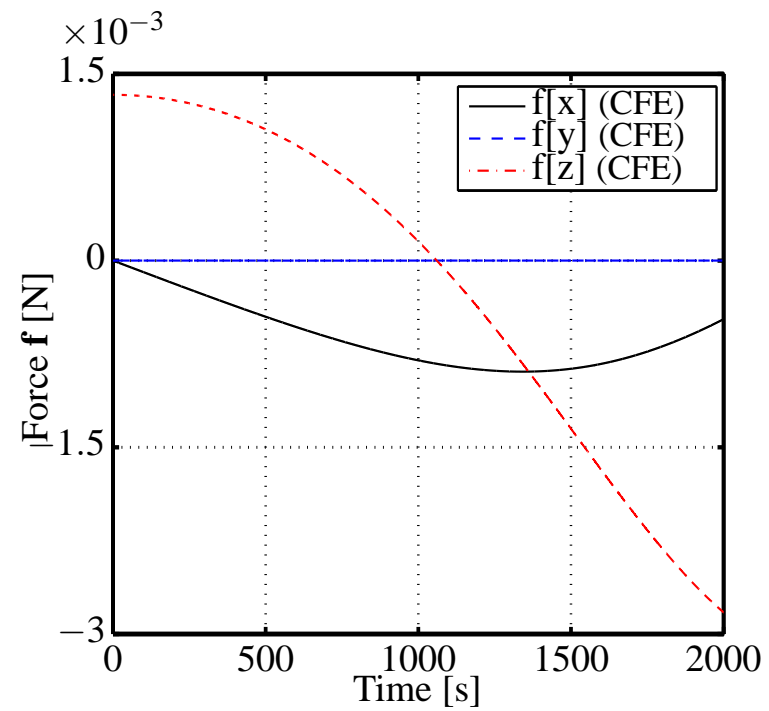

(a) Constraint forces at joint during connected motion with CFE methodology, in all ECI directions $i=x, y, z$.

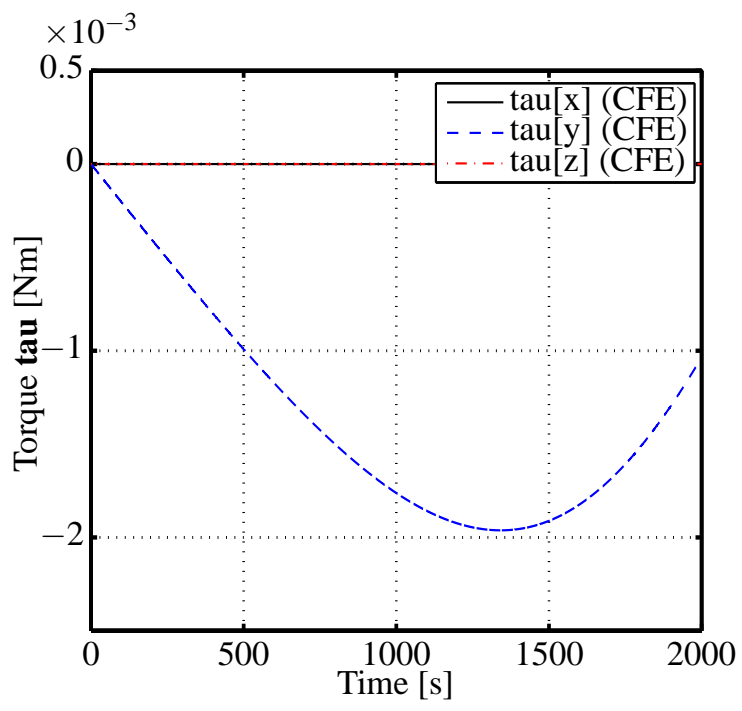

(c) Constraint torques at joint during connected motion with CFE methodology, in all ECI directions $i=x, y, z$.

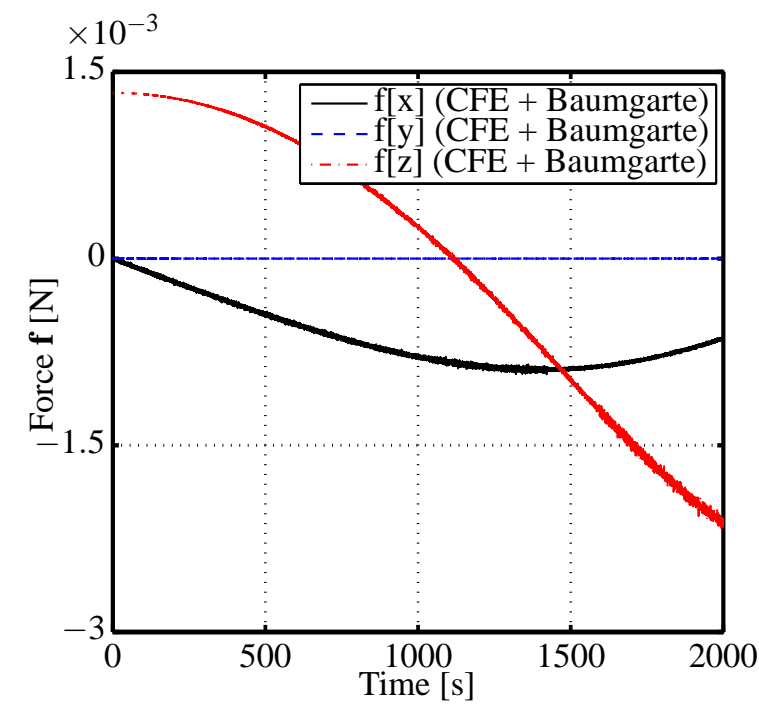

(b) Constraint forces at joint during connected motion with CFE methodology plus Baumgarte stabilization with $\eta=2$, in all ECI directions $i=x, y, z$.

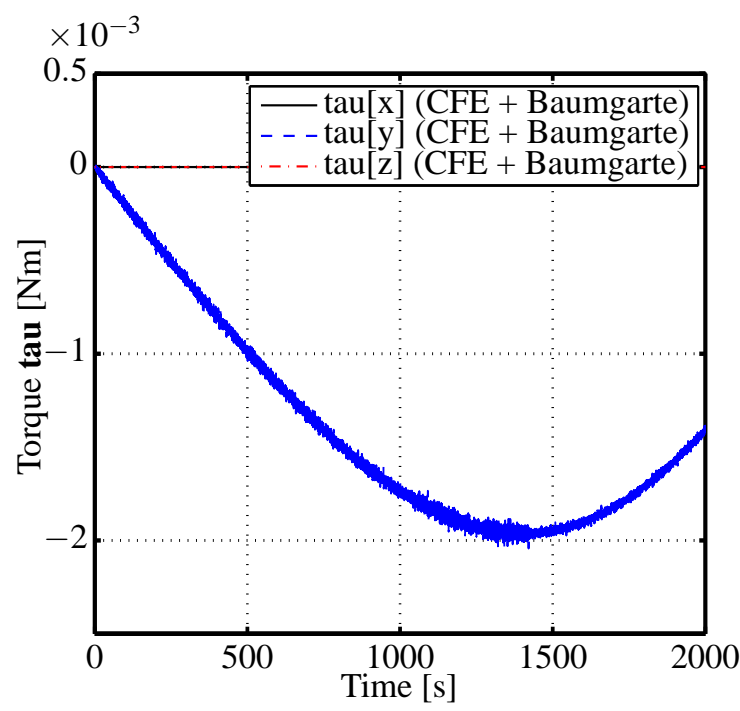

(d) Constraint torques at joint during connected motion with CFE methodology plus Baumgarte stabilization with $\eta=2$, in all ECI directions $i=x, y, z$.

Figure 4: Case Study A results: constraint forces and torques at joint during connected motion.

(d), 5-(b), 5-(d), meaning more computational time and effort.

Case Study II, as outlined in the last section, accounts for the study of absolute- and relative- position, velocity, and acceleration, respectively, between the two bodies from a multi-stage separation dynamics practical scenario. In here, the 'release device' simulated by a linear charge model has been augmented with an 'impulsive device' in parallel simulated by a kick-off spring model in order to simulate such a separation mechanism between the two bodies at their time of release from each other.

In this respect, Figure 6 presents the bodies' relative position $\operatorname{rrel}[i]$, velocity $\operatorname{vrel}[i]$, and acceleration $\operatorname{arel}[i]$ along the ECI orbital flight direction $i=y$ (which is valid only for such a very small time frame) during the connected motion (first 10 seconds), and during their subsequent separation (last 10 seconds). Figure 6 also presents a zoom of the small time window just around the separation command.

Results of this separation scenario shows the corre- 
$\times 10^{-7}$

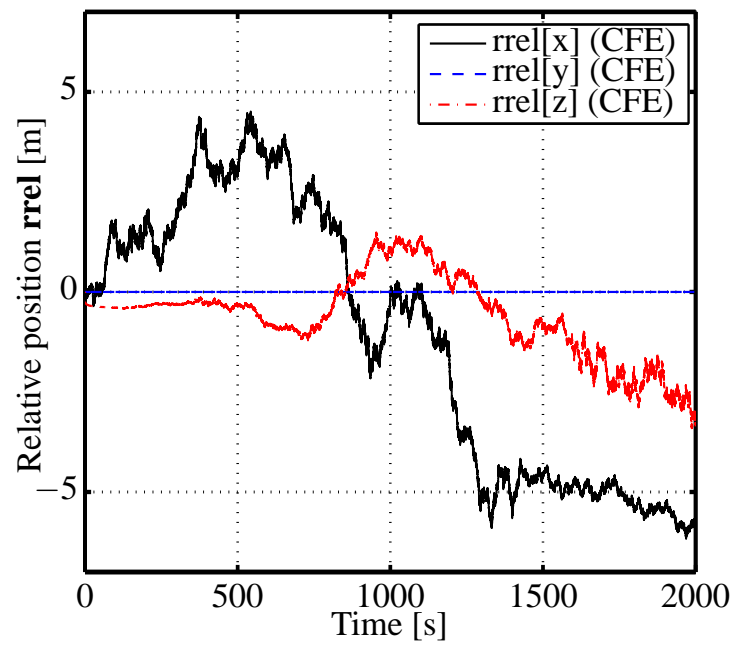

(a) Relative joint position during connected motion with CFE methodology, in all ECI directions $i=x, y, z$.

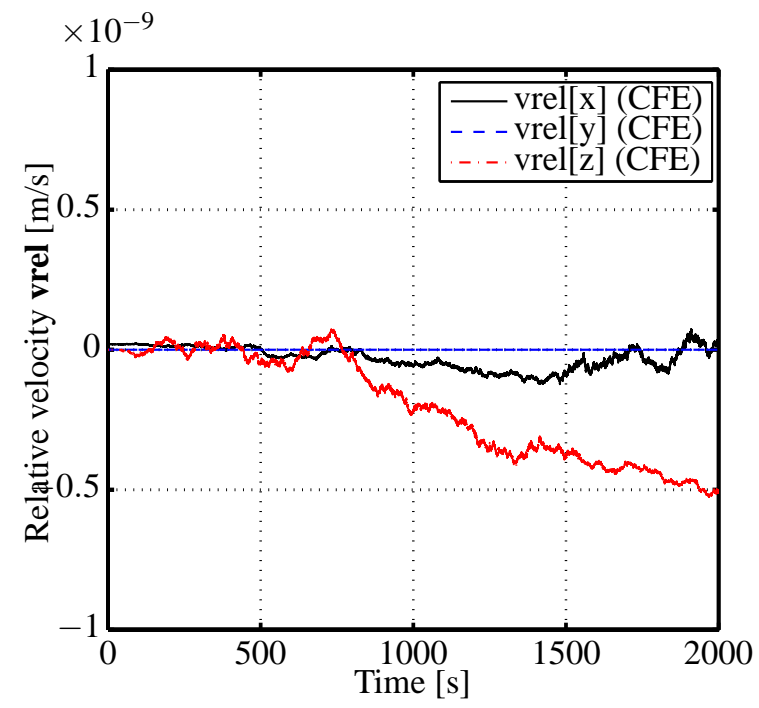

(c) Relative joint velocity during connected motion with CFE methodology, in all ECI directions $i=x, y, z$.

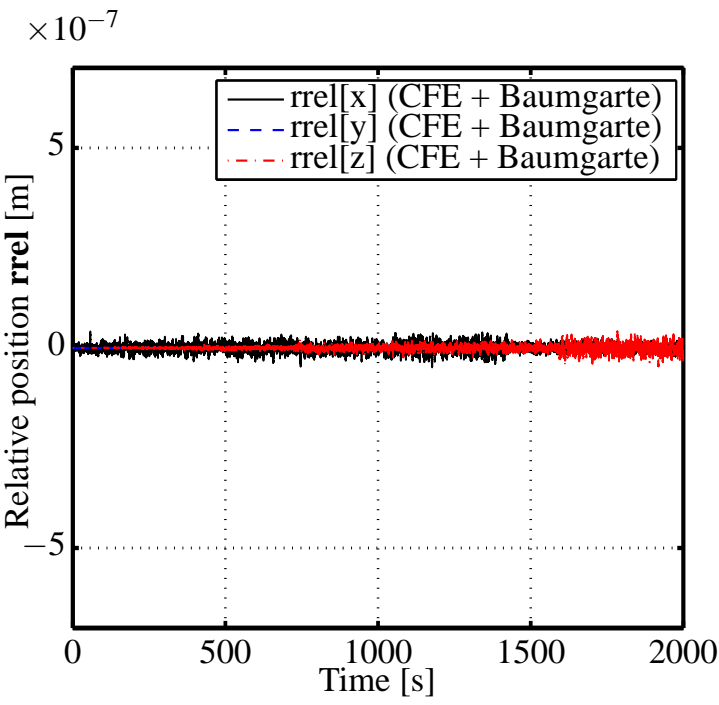

(b) Relative joint position during connected motion with CFE methodology plus Baumgarte stabilization with $\eta=2$, in all ECI directions $i=x, y, z$.

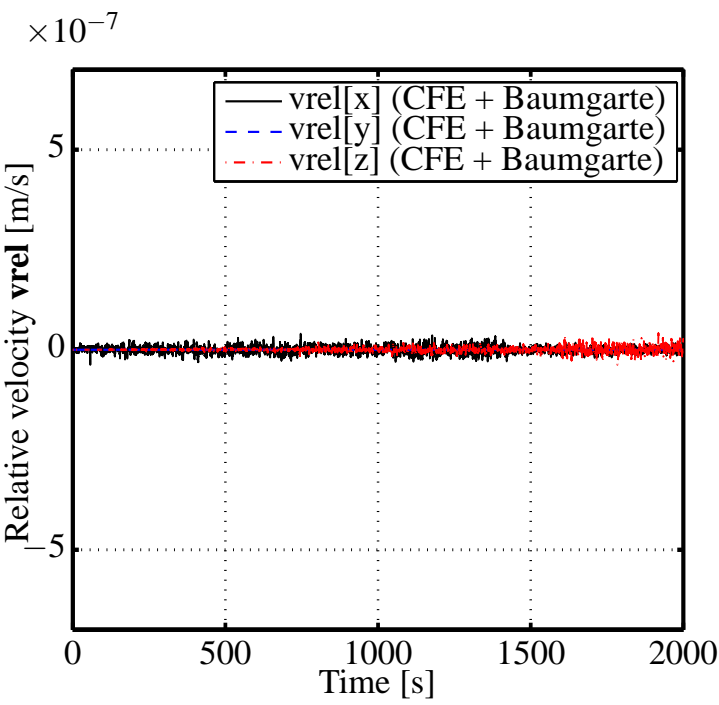

(d) Relative joint velocity during connected motion with CFE methodology plus Baumgarte stabilization with $\eta=2$, in all ECI directions $i=x, y, z$.

Figure 5: Case Study A results: relative joint position and velocity during connected motion, in all ECI directions $i=x, y, z$.

sponding relative states of the composite up until separation command and then their subsequent independent flight. Once again, the benefit and ease of use of the Modelica implementation of the CFE methodology is evidenced during the connected flight of the composite, since constraint forces and torques are automatically computed and applied to the system. At separation, the relative states suggest an impulsive behaviour due to the kick-off spring separation mechanism model. This model releases a pre-compressed force stored in a replaceable spring-damper model, ev- idencing good correspondence with the physics behind separation. Such devices result in impulsive forces applied to the two-body system. This in turn causes a change in relative velocity and therefore, a successful physical separation of the system.

\section{Conclusion}

The objective of this paper was to present an object-oriented and equation-based acausal modeling approach to launch vehicle stage separation dynamics 
with ModelicA. The aim is to develop an integrated approach for end-to-end launch vehicle trajectory simulation within a single environment.

Based on the Constraint Force Equation (CFE) methodology, two case studies to evaluate the proposed approach were considered. The scenario under study consisted of two bodies -representing a generic launcher stage and its payload-prior, during, and after their separation in orbital flight motion.

Results demonstrated that the approach, mainly thanks to the acausal and equation-based modeling features of the MoDELICA language, corresponds very well with the physics behind separation while providing easiness of implementation within a single environment such as DyMOLA. The method computes and applies constraint loads automatically during joint motion and removes them accordingly at separation time, all in consistency with the CFE methodology.

A disadvantage for long simulation periods of joint body motion is the necessity to keep the drift within physical boundaries, hence requiring a stabilization method. This in turn increases chattering and computational time and effort, thus resulting in a trade-off to consider for the task at hand. Validation studies are left to future work.

\section{Acknowledgements}

This work corresponds to DLR Institute of System Dynamics and Control activities of the ESA study Upper Stage Attitude Control Design Framework (USACDF), led by Astrium GmbH as part of Europe's Future Launchers Preparatory Program (FLPP).

\section{References}

[1] Decker, J. P. Experimental Aerodynamics and Analysis of the Stage Separation of Reusable Launch Vehicles. NASASP-148, January 1967.

[2] Decker, J. P., and Gera, J. An Exploratory Study of ParallelStage Separation of Reusable Launch Vehicles. NASA TN D-4765, October 1968.

[3] Baumgarte, J. Stabilization of Constraints and Integrals of Motion in Dynamical Systems. Computer Methods in Applied Mechanics and Engineering, Vol. 1, No. 1, 1972, pp. 1-16.

[4] Decker, J.P., and Wilhite, A. W. Technology and Methodology of Separating Two Similar Size Aerospace Vehicles Within the Atmosphere.AIAA Paper 1975-29, Jan. 1975.
[5] Bauer, G.L., Cornick, D.E., and Stevenson, R. Capabilities and Applications of the Program to Optimize Simulated Trajectories (POST). NASA CR-2770, 1977.

[6] Ascher, U.M., Chin, H., Petzold, L.R., and Reich, S. Stabilization of constrained mechanical systems with DAEs and invariant manifolds. Journal of Mechanics of Structures and Machines, Vol. 23, 1994, pp 135-157.

[7] Elmqvist, H., Mattson, S.E., and Otter, M. Modelica - An International Effort to Design an Object-Oriented Modeling Language. Summer Computer Simulation Conference, pp. 333-339, 1998, ISBN 1-56555-149-4.

[8] Mattson, S.E., Elmqvist, H., and Otter, M. Physical system modeling with Modelica. Control Engineering Practice, v. 6, pp. 501-510, 1998.

[9] Otter, M., Elmqvist, H., and Mattsson, S.E. The New Modelica Multibody Library. Proceedings of the 3rd International Modelica Conference, Linköping, 2003, pp 311-330.

[10] Tartabini, P. V., Bose, D. M., McMinn, J. D., Martin, J. G., and Strovers, B. K. Hyper-X Stage Separation Trajectory Validation Studies. AIAA Paper 2003-5819, August 2003.

[11] Murphy, K.J., Buning, P.G., Pamadi, B.N., Scallion, W.I., and Jones, K.M. Status of Stage Separation Tool Development for Next Generation Launch Vehicle Technologies. AIAA Paper 2004-2595.

[12] Pamadi, B.N., Neirynck, T. A., Covell, P.F., Hotchko, N.J., and Bose, D.M. Simulation and Analyses of Staging Maneuvers of Next Generation Reusable Launch Vehicles. AIAA Paper 2004-5185.

[13] Pamadi, B.N., Hotchko, N.J., Jamshid Samareh, Covell, P.F., and Tartabini, P.V. Simulation and Analyses of MultiBody Separation in Launch Vehicle Staging Environment. AIAA Paper 2006-8033.

[14] Pamadi, B.N., Neirynck, T. A., Hotchko, N.J., Scallion W.I., Murphy, K.J., and Covell, P.F. Simulation and Analyses of Stage Separation of Two-Stage Reusable Launch Vehicles. Journal of Spacecraft and Rockets, Vol. 44, No. 1, January-February 2007, pp 66-80.

[15] Källdahl M. Separation Analysis with OpenModelica. Student thesis, Linköping University, Department of Electrical Engineering, Institutionen fr̉ systemteknik, Sweden, 2007.

[16] Toniolo, M., Tartabini, P.V., and Pamadi, B.N. Constraint Force Equation Methodology for Modeling Multi-Body Stage Separation Dynamics. 46th AIAA Aerospace Sciences Meeting and Exhibit, 2008, 10.2514/6.2008-219.

[17] Tartabini, P.V., Roithmayr, C.M., Toniolo, M.D., Karlgaard, C.D., and Pamadi, B. N. Modeling Multibody Stage Separation Dynamics Using Constraint Force Equation Methodology. Journal of Spacecraft and Rockets, Vol. 48, No. 4, July-August 2011, pp 573-583.

[18] Pamadi, B.N., Tartabini, P.V., Toniolo, M.D., Roithmayr, C.M., Karlgaard, C.D., and Samareh, J.A. Application of Constraint Force Equation Methodology for Launch Vehicle Stage Separation. Journal of Spacecraft and Rockets, Vol. 50, No. 1, January-February 2013, pp 191-205. 


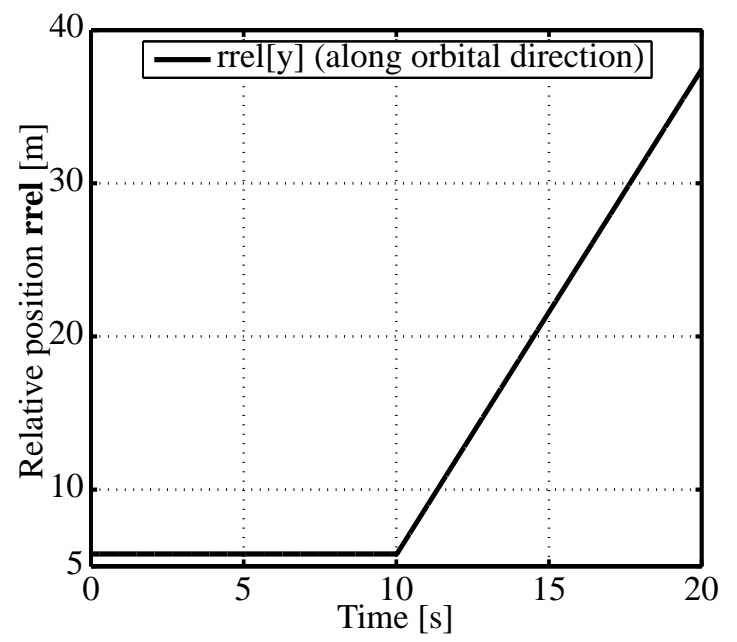

(a) Relative position between bodies $A$ and $B$. The initial relative position $(5.8 \mathrm{~m})$ corresponds to the fixed distance between the bodies center of masses during joint motion.

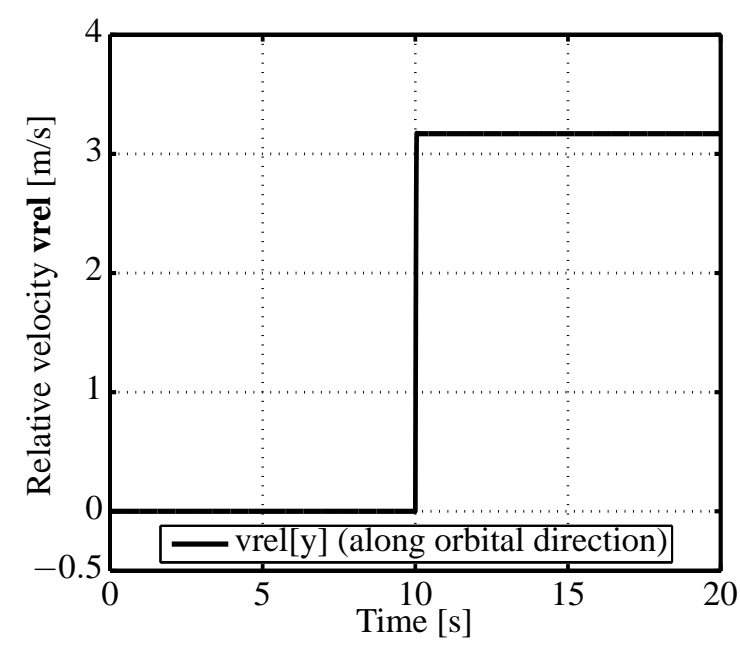

(c) Relative velocity between bodies $A$ and $B$.

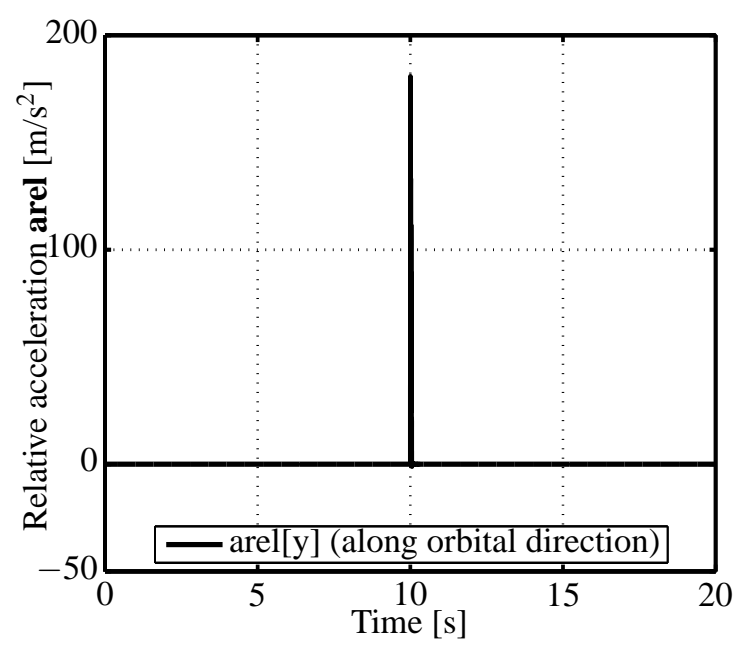

(e) Relative acceleration between bodies $A$ and $B$.

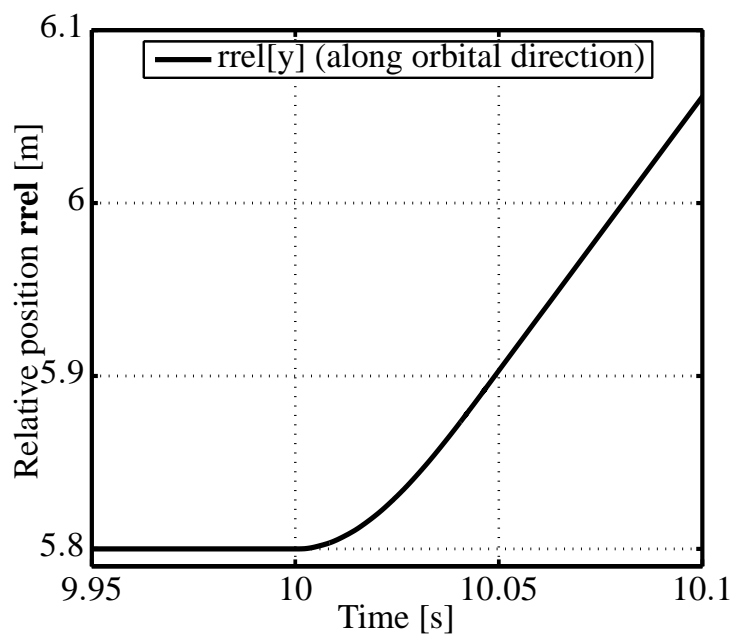

(b) Same as (a) with a close view around time of separation.

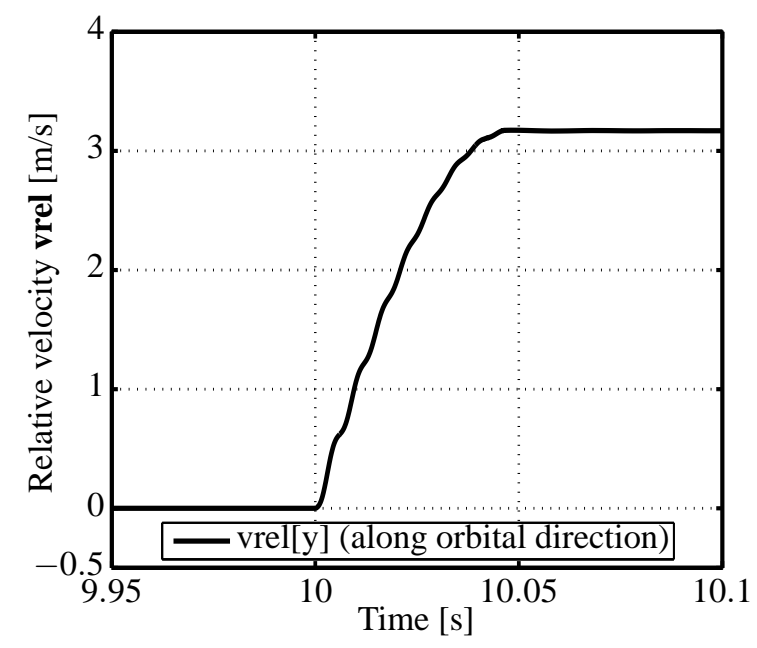

(d) Same as (c) with a close view around time of separation.

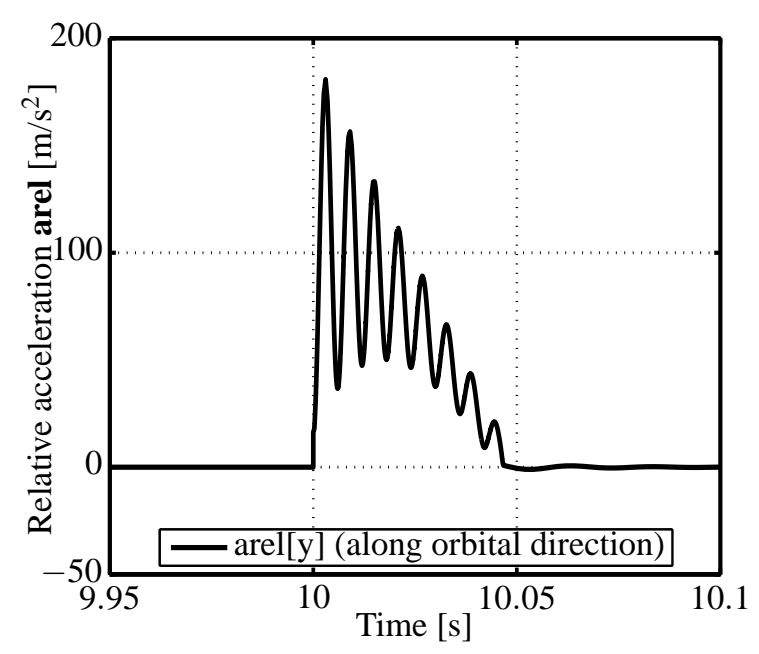

(f) Same as (e) with a close view around time of separation.

Figure 6: Case Study B results: Relative position, velocity, and acceleration from a kick-off separation scenario along orbital flight direction $i=2$. Ignition / separation command at $t=10 \mathrm{~s}$. 